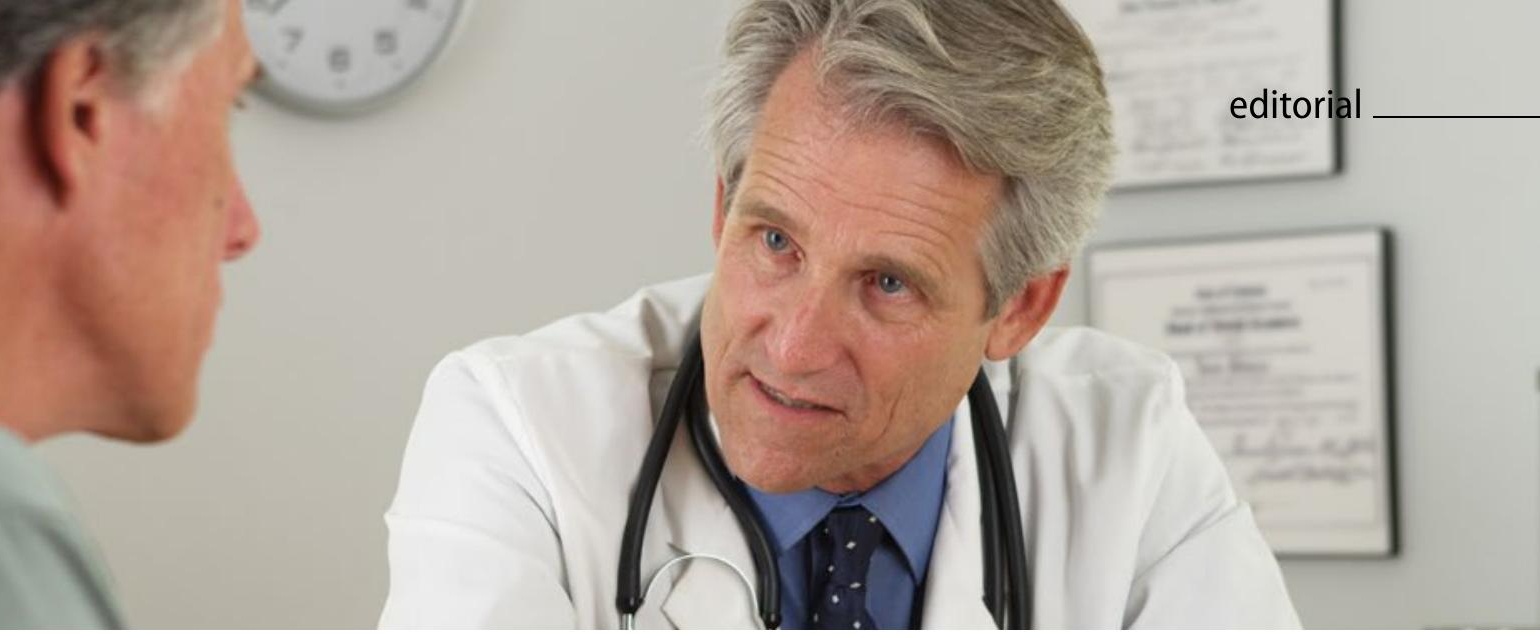

Krebsberatung durch Krebsgesellschaften

\title{
Mehr Anerkennung verdient!
}

Menschen, die mit der Diagnose Krebs konfrontiert werden, Betroffene wie Angehörige, haben Fragen über Fragen. Jedoch dann, wenn Unsicherheit, ja Not am größten sind, fehlt oft der verständnisvolle und kompetente Gesprächspartner. Selbstverständlich ist das Gespräch mit dem behandelnden Arzt der richtige Ort und Zeitpunkt, Antworten zu erhalten. Aber: Zu Beginn des Krebsverdachtes bleibt angesichts der Fülle diagnostischer und therapeutischer Maßnahmen kaum Zeit dafür, sich über die eigenen Fragen klar zu werden und alle Informationen auch zu verstehen. Ähnlich kritisch ist die Phase bei einem Rückfall, einer Metastasierung, wenn man mit der Endlichkeit des Daseins konfrontiert wird und man sich der Angst kaum erwehren kann. Da helfen Angehörige, die mit zuhören, aber diese haben, gleich welchen Geschlechtes, einen signifikant höheren Angstspiegel als die Betroffenen selber und sind dann überfordert [Grov EK et al. Ann Oncol. 2005;16(7):1185-91].

In einer Vielzahl von Studien zeigte sich, dass für 20-60\% der Betroffenen, situativ bedingt auch sehr viel häufiger, das ärztliche Gespräch nicht ausreicht [Higginson IJ, Costantini M. J Clin Oncol. 2002;20(17):3674-82; Kleeberg UR et al. Support Care Cancer. 2008;16(8):947-54]. Es gibt eine Kluft zwischen dem Bedarf, wie er von der Ärzteschaft bestenfalls nur teilweise erfüllt werden kann, und den Bedürfnissen der Betroffenen und ihrer Angehörigen.

\section{Essentielle Abhilfe durch Krebsgesellschaften}

Hier kommt den Beratungsstellen der 16 Landeskrebsgesellschaften der Deutschen Krebsgesellschaft (DKG) große Bedeutung zu. Seit den 1950er-Jahren werden täglich von DKG-Experten Beratungen und Aktionen zu den Themen Prävention, Krankheit, Behandlung und Krankheitsbewältigung durchgeführt: Laut DKG-Jahresbericht 2017 erfolgten im Jahr 2015 61.738 individuelle Beratungen (Einzelberatung, Paarberatung, Familienberatung). Hinzu kamen 379 Kurse und Gruppenangebote mit kreativen Inhalten sowie 950 Informationsveranstaltungen, ergänzt durch Broschüren und Selbsthilfeinitiativen. Damit sind die Landeskrebsgesellschaften mit Abstand der größte Anbieter von Krebsberatungen in Deutschland. Mit 60-70\% machen Krebspatienten den größten Teil der Ratsuchenden aus, ca. 30-40\% sind Angehörige und ein kleiner Anteil besteht aus professionellen Helfern oder Interessierten. Die Beratungen sind persönlich, zeitnah, niederschwellig und

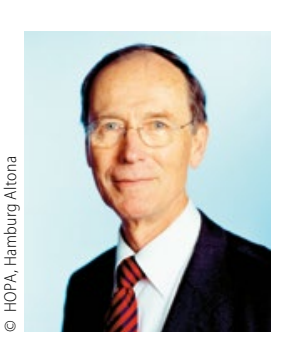

Prof. Dr. Ulrich R. Kleeberg

Hämatologisch-onkologische

Praxis Altona, Tagesklinik Struensee-Haus,

Hamburg

urkleeberg@hopa-hamburg.de

finden kostenfrei statt. Sie sind situativ auf den Ratsuchenden abgestimmt und können so häufig stattfinden, wie der Ratsuchende es wünscht bzw. es notwendig ist (in der Regel bis zu fünfmal). Sie sind sowohl bezüglich der Häufigkeit als auch der Inhalte entsprechend des Patientenbedarfs „maßgeschneidert“.

Die öffentliche Anerkennung, ausgedrückt auch in der Finanzierung durch die Krankenkassen, die Sozialversicherungsträger, die staatlichen Gesundheitsämter, beschränkt sich immer noch auf Willensbekundungen - und das trotz des Nationalen Krebsplanes des Bundesgesundheitsministeriums, der gemeinsam mit der DKG und den Krankenkassen vor fast zehn Jahren verabschiedet worden ist [Richter-Kuhlmann E. Dtsch Arztebl. 2017;114(24):A-1168/ B-976/C-954; siehe Handlungsfeld 4: Patientenorientierung]. Die qualitativ hoch bewertete Arbeit der Krebsberatungsstellen wird stattdessen entscheidend durch Spenden getragen, in Hamburg unter dem Motto „Von Hamburgern für Hamburger“. Sie erlaubten es der Hamburger Krebsgesellschaft auch, einen Stiftungslehrstuhl für Palliativmedizin einzurichten und zu finanzieren.

Die Krebsgesellschaften unterstützen Betroffene nicht nur in der ganzen Breite des Krankheitsgeschehens - von der Erkrankung selbst bis zu den somatischen und psychosozialen Folgen. Sie leisten zudem viel für die Qualitätssicherung ihrer Arbeit, etwa durch „standard operating procedures“ (SOP) oder eine systematische Basis- und Verlaufsdokumentation. Es ist wieder einmal an der Zeit, diese vielfältigen und großartigen Bemühungen der Krebsgesellschaften etwas mehr ins Bewusstsein der Öffentlichkeit zu rücken - wenigstens in das der Ärzte.

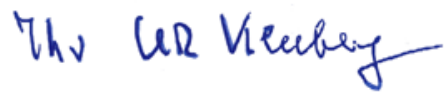

